\title{
Expression of VEGF and collagen using a latex biomembrane as bladder replacement in rabbits
}

André Luís Alonso Domingos, Sérgio Britto Garcia, José de Bessa Jr., Marcelo Ferreira Cassini, Carlos

Augusto Fernandes Molina, Silvio Tucci Junior

Department of Surgery, Division of Urology, Ribeirão Preto Medical School - University of Sao Paulo Ribeirao Preto, SP and Department of Experimental Surgery, Anhanguera-Uniderp University, Campo Grande, MS, Brazil

\section{ABSTRACT}

Objective: To investigate the VEGF expression and collagen deposition using a latex biomembrane as bladder replacement in rabbits.

Materials and Methods: After partial cystectomy, a patch of a non-vulcanized latex biomembrane $(2 \times 2 \mathrm{~cm})$ was sewn to the bladder of rabbits with 5/0 monofilament polydioxanone sulfate sutures in a watertight manner. Groups of 5 animals were killed at 15, 45 and 90 days after surgery and the bladder was removed. Sections of $5 \mu \mathrm{m}$ were cut and stained with picrosirius-red in order to estimate the amount of extracellular matrix in the graft. To confirm the presence of VEGF in tissues, protein expression was determined by immunohistochemistry.

Results: No death, urinary leakage or graft extrusion occurred in any group. All bladders showed a spherical shape. A progressive reduction in the amount of collagen occurred in the graft area and was negatively and linearly correlated with time ( $\mathrm{p}<$ 0.001). VEGF expression was higher in grafted areas when compared to controls at 15 and 45 days after surgery and decreased with time $(\mathrm{p}<0.001)$.

Conclusion: The latex biomembrane as a matrix for partial bladder replacement in rabbits promotes temporary collagen deposition and stimulates the angiogenic process.
ARTICLE INFO

\section{Key words:}

Latex; Collagen;

Urinary Tract;

Tissue-engineering

Int Braz J Urol. 2012; 38: 536-43

Submitted for publication:

January 16, 2012

Accepted after revision:

May 22, 2012

\section{INTRODUCTION}

Bladder augmentation or replacement has the following objectives: reduction of intravesical pressure, improvement of urinary continence and preservation of urinary tract function. The use of gastrointestinal segments remains, even today, the technique most frequently employed for this purpose. Augmentation cystoplasty is currently used in the treatment of approximately 5\% to 25\% of patients with spina bifida and $13 \%$ to $50 \%$ of those with bladder exstrophy (1). However, the potential complications of the use of a portion of the gas- trointestinal tract to perform augmentation cystoplasty have been well characterized. They include the development of intestinal and urinary fistulae, bladder stones, production of mucus, spontaneous bladder perforation, electrolyte imbalance, and carcinogenesis (2-4). Autoaugmentation and ureterocystoplasty have also been advocated as a solution in subsets of patients, but the former does not elicit long-term consistent results and the latter depends on the presence of a nonfunctioning kidney unit (5).

Bladder tissue engineering attempts to restore native functions of impaired bladders using biodegradable matrices that serve as templates 
to guide and orient host tissue regeneration as well as harbor environmental signals to promote proper tissue growth. Development of biodegradable scaffolds seems to be more appropriate because they would allow the appropriate time for host tissue regeneration with dissolution before a foreign body reaction (6). In recent years, the porcine small intestinal submucosa (SIS) and the bladder acellular matrix graft (BAMG) have been used frequently in experimental studies. They act as biodegradable materials that allow urothelial and smooth muscle regeneration (7). However, the SIS and BAMG are xenogeneic materials and need advanced technical preparation.

Previous experimental studies with the natural latex biomembrane have proved that it is a biodegradable material of easy preparation and handling, encouraging tissue neoformation $(8,9)$. On this basis, we were interested in investigating collagen development and angiogenesis using this latex graft. The aim of the present study was to investigate vascular endothelial growth factor (VEGF) expression and collagen deposition in bladder replacement using the latex biomembrane in rabbits.

\section{MATERIALS AND METHODS}

A total of 15 adult male New Zealand rabbits weighting 3.0-3.4 kg underwent bladder augmentation with the latex biomembrane. This project was approved by the Animal Research Committee of our Institution. Groups of five animals were sacrificed at 15 (group A), 45 (group B) and 90 (group C) days after grafting. As controls, in each rabbit we excised a full-thickness bladder fragment far from the grafted area.

\section{Membrane preparation and Surgical Technique} Matrix preparation followed a previously published technique $(8,9)$. The animals were anesthetized with an intramuscular injection of ketamine $(35 \mathrm{mg} / \mathrm{kg}$ ) and xylazine $(5 \mathrm{mg} / \mathrm{kg})$. A partial cystectomy $(4.0 \mathrm{~cm} 2-2.0 \times 2.0 \mathrm{~cm})$ was performed through a median laparotomy. A latex biomembrane patch of the same area was grafted to the remaining host bladder with continuous 5/0 monofilament absorbable polydioxanone sulfate suture as described in our previous study (9). After surgery, enrofloxacyn $(9 \mathrm{mg} / \mathrm{kg})$ was administered subcutaneously to all animals daily for 2 days. The clinical condition of all animals was evaluated daily from surgery to sacrifice. Animals were sacrificed with an intravenous injection of pentobarbital (60 mg/kg).

\section{Histology and Immunohistochemistry}

Formalin-fixed specimens from the grafted and control areas of the bladder were embedded in paraffin. Sections of $5 \mu \mathrm{m}$ were cut and stained with picrosirius-red in order to estimate the amount of extracelullar matrix in the graft.

An immunohistochemical method for VEGF labeling was used to assess angiogenesis. Antibody expression was determined by antigenantibody reaction followed by development of the reaction with a marker visible under the microscope. The immunohistochemical reactions were evaluated by light microscopy and immunoreactivity was considered to be positive when staining was detected in the cell cytoplasm or nucleus. The labeling index was determined as the ratio between the total number of cells and the number of deeply stained cells.

To confirm the presence of VEGF in tissues, protein expression was determined by immunohistochemistry using the VEGFR-3 marker (Novocastra ${ }^{\circledR}$ ), clone KLT9, at a 1:200 dilution.

The number of cells with positive staining for VEGF was estimated with an Axio Cam camera (Zeiss, Germany) and the Axiovision 4.6 software (Zeiss, Germany).

Ten random microscope fields were examined for histological/immunohistochemical evaluation. Cells were counted at 400X magnification by two different pathologists in a blind manner. A semi-quantitative analysis was performed and the results are reported as the percentage of positive cells in total tissue as shown in Table- 1 .

\section{Statistical Analysis}

Data are reported as medians and range. Data regarding the treated and control areas were compared by the Wilcoxon test. The relationships 
between groups were analyzed by analysis of variance (Kruskal-Wallis test), followed by the Dunn test to compare individual pairings. Statistical analysis was performed using the GraphPad Prism 5.00 software (San Diego, CA, USA) and p values < 0.05 were considered to be statistically significant.

Table 1 - Semi-quantitative scores: percentage of collagen deposition and VEGF expression.

\begin{tabular}{lcc}
\hline Degree & Collagen & VGEF \\
\hline Score 0 & Absent & Absent \\
Score $1(+)$ & $<25 \%$ & $<25 \%$ \\
Score 2 $(++)$ & $25 \%$ to $50 \%$ & $25 \%$ to $50 \%$ \\
Score 3 $(+++)$ & $50 \%$ to $75 \%$ & $50 \%$ to $75 \%$ \\
Score $4(++++)$ & $>75 \%$ & $>75 \%$ \\
\hline
\end{tabular}

\section{RESULTS}

All rabbits survived the procedures and were able to void spontaneously after the operation and no one exhibited urinary leakage. A bladder stone was found in one rabbit (6.6\%) after 45 days.

Macroscopic evaluation

All bladders showed a spherical shape.

On the 15th postoperative day, blood ves- sels were visible around the grafted area. After a longitudinal incision of the bladder, the presence of the latex matrix was easily identified since it was almost intact.

On the 45th day after surgery, the graft was almost integrated to the host tissue of the native bladder. The bladder wall was thicker, mainly around the latex membrane. One bladder stone $(1.5 \times 2.0 \mathrm{~cm})$ was observed in one rabbit $(6.6 \%)$.

Ninety days after surgery, there was a decrease in bladder wall hypertrophy and the graft was indistinguishable from the normal host bladder on both the inner and outer surfaces (Figure-1). There was blood vessel reduction around the grafted area.

\section{Microscopic evaluation}

On the 15th postoperative day the luminal surface of the latex matrix was still uncovered by the urothelium. A fibrovascular reaction was present, with rare fibroblasts and a moderate amount of inflammatory cells, mainly macrophages. Forty-five days after surgery there was diffuse epithelial and smooth muscle hyperplasia on the graft. At 90 days there was a reduction of epithelial and muscle hyperplasia, and the urothelium was similar to that of the native bladder. At that time, the smooth muscle layers were well organized and oriented in a direction similar to that of native bladder muscle layers (Figure-2).

\section{Collagen deposition}

The non-grafted areas of the bladder (control) showed the presence of well compacted col-

Figure 1 - Macroscopic evaluation of the latex biomembrane: (A) spherical architecture (exterior); (B) border between the native and new tissues (interior).

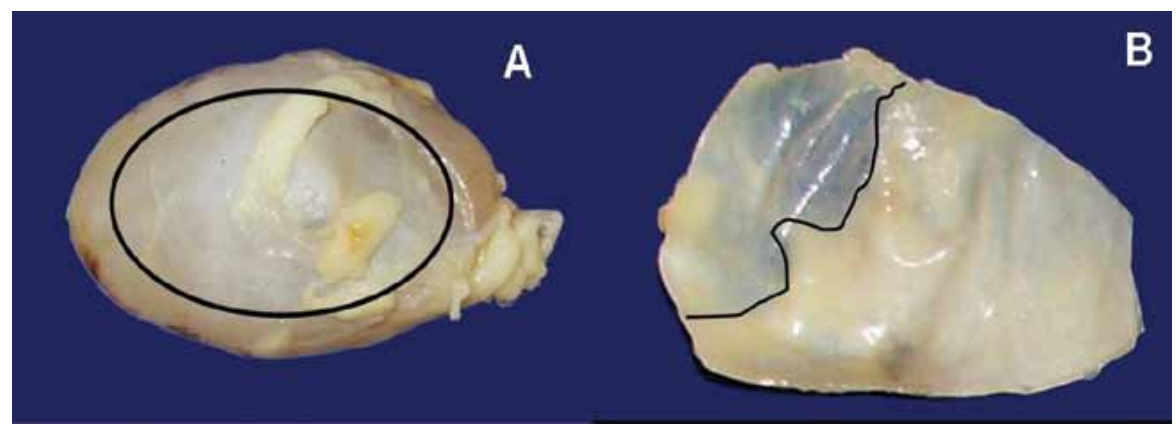


Figure 2 - Microscopic evaluation: (A) normal epithelium in non grafted area. (B) 15 days after surgery: note the presence of blood vessels (black arrows). (C) 45 days after surgery: epithelium recovering the grafted area (red arrow). (400X). (Hematoxylin and Eosin - HE).

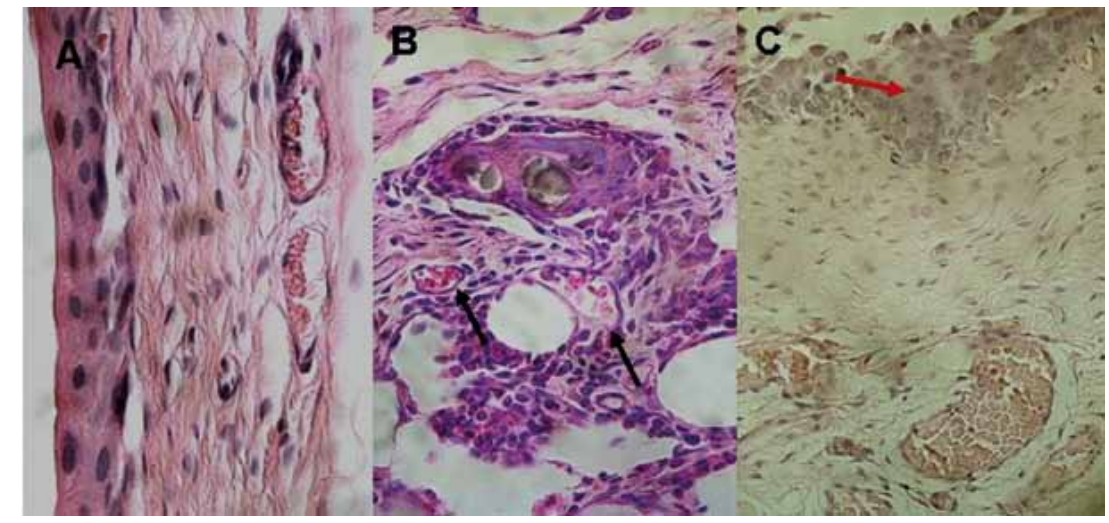

lagen fibers, mainly in the submucosal layer, with lower density in the muscle layer. Fifteen days after the implant there was an overall increase in the amount of collagen, with high density on the muscle plans and in the submucosa. Three months after the graft, there was a reduction in the amount of collagen on the plans corresponding to the muscle layers and a tendency to greater concentration in the submucosa, similar to control (Figure-3).

Collagen expression differed significantly between the bladders examined after the latex membrane implant and their respective controls (Table-1). A progressive reduction in the amount of collagen occurred in the graft area and was negatively and linearly correlated with time $(\mathrm{p}<0.001)$ (Spearman $\mathrm{r}=-0.88[-0.69$ to -0.97$]$ 95\% CI.

\section{VEGF Expression}

Group A animals (15 days) showed intense positive labeling for VEGF in undifferentiated cells close to the epithelial basement membrane and around the fragments of the latex membrane on the

Figure 3 - Evaluation of collagen. I: control; II: 15 days after the graft; III: 90 days after the graft. Picrosirius-red (Magnification 400X). C (colagen), E (epithelium). (400X).

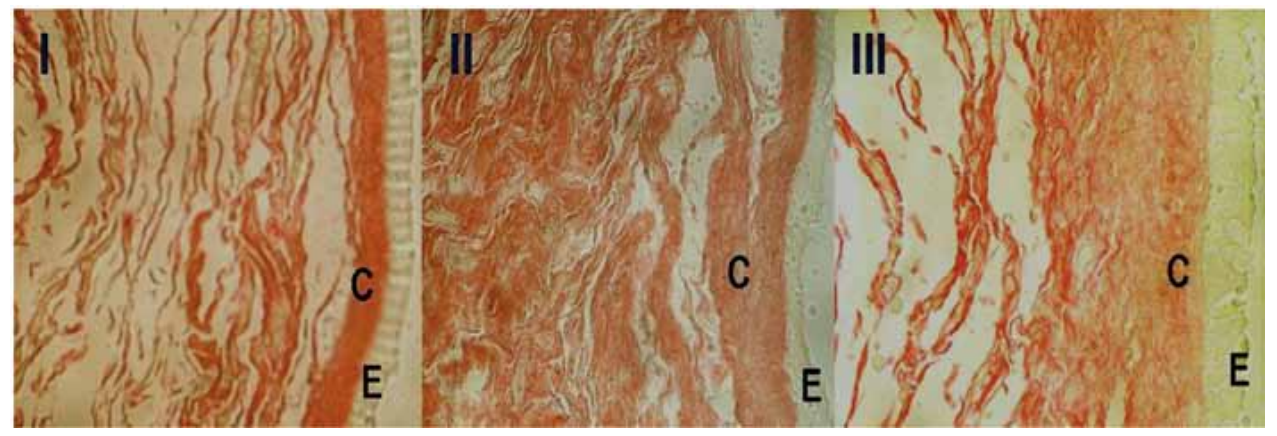


bladder wall (Figure-4). The expression of the angiogenesis marker differed significantly between the experimental group and control, at different times (Table-2).

As also observed for collagen, a progressive reduction in VEGF labeling occurred along time ( $\mathrm{p}$ $<0.001$ ) (Spearman $\mathrm{r}=-0.92[-0.75$ to -0.97$]$ 95\% CI. These data are illustrated in Table- 2 .

\section{DISCUSSION}

Previous experimental studies with the latex biomembrane have shown that it allows the ingrowth of muscle cells and induces a temporary inflammatory response, mainly a TH2 lymphocyte immune response (9). The ideal material for bladder replacement or enlargement should allow the

Figure 4 - VEGF expression in grafted and non grafted areas over the time. (A) - non grafted area. (B) - grafted area 15 days after surgery: higher VEGF expression (black arrows) and remaining latex membrane (red arrows). (C) - grafted area 90 days after surgery: note the reduction of VEGF expression. (400x).

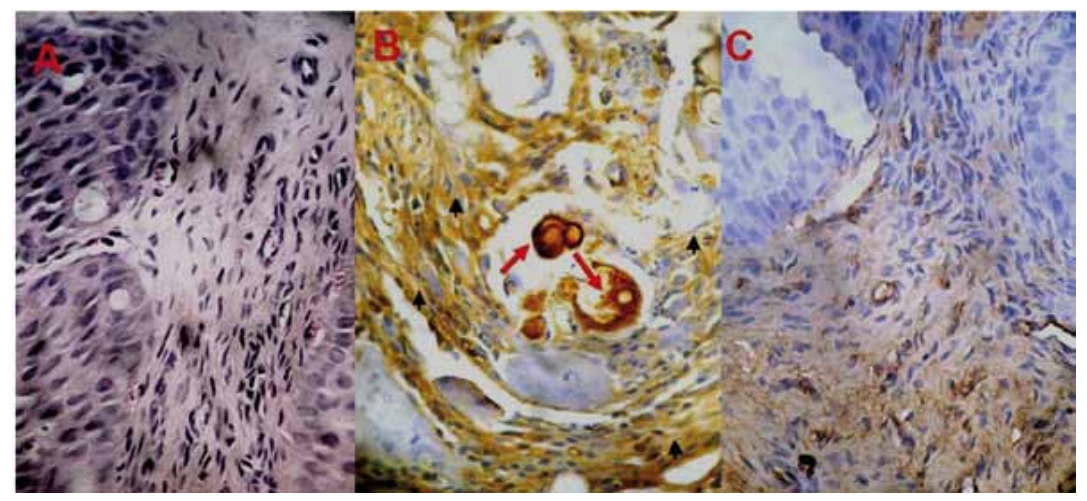

Table 2 - Scores of expression of collagen and VEGF in the groups (experimental and control) at different times of evaluation. Data are expressed as median and range.

\begin{tabular}{lccc}
\hline & Control & Experimental & p-value \\
\hline Collagen deposition & & & \\
$15^{\text {th }}$ day & $1(1-1)$ & $4(3-4)$ & $<0.001$ \\
$45^{\text {th }}$ day & $1(1-1)$ & $3(2-3)$ & $<0.001$ \\
$90^{\text {th }}$ day & $1(1-1)$ & $2(1-2)$ & 0.016 \\
VEGF expression & & & $<0.001$ \\
$15^{\text {th }}$ day & $1(1-1)$ & $4(3-4)$ & $<0.001$ \\
$45^{\text {th }}$ day & $1(1-1)$ & $3(2-3)$ & $2(1-2)$ \\
$90^{\text {th }}$ day & $1(1-1)$ & 0.016 \\
\hline
\end{tabular}


progressive growth of all components of the normal bladder wall preserving their mechanical and functional properties $(7,10)$.

When present inside the wound, fibroblasts act on the synthesis, deposition and remodeling of the extracellular matrix (ECM), performing a new protein synthesis function, especially of collagen. The balance between ECM synthesis and degradation results in the remodeling of the structure of connective tissue, a fundamental process in wound repair (11). The repair of tissue damaged by surgical resection, by wounds or by other chronic lesions can be divided into two processes, i.e., regeneration and scarring. The former results in the restitution of lost tissues, and the latter involves collagen deposition and scar formation.

The amount of collagen is an essential component of the mechanical properties of bladder grafts, conferring strength and maintaining the expansibility of the organ. It has been reported that the amount of collagen in animals submitted to bladder expansion with an acellular graft was significantly greater than in bladders expanded with a graft previously seeded with autologous cells (12), suggesting that the acellular graft used (polyglycolic acid) without a previous cell implant may lead to a foreign body reaction. Conversely, a study assessing the acellular matrix (BAMG) revealed that the amount of collagen, especially type I, was more marked during the first 15 days of graft implant, accompanying the intensity of the inflammatory process, and was up to 2.5 times greater than in the native bladder used as control. However, 3 months after surgery the density of collagen fibers in the grafts were the same as in the normal bladder (13).

The tissue responses (cellular and humoral) that signal regeneration or scar formation are intimately related and may be detected at the beginning of the natural process of injury and inflammation. For this reason, this difference should not be attributed exclusively to the production of collagen or to the inflammatory process (14). Animal models have demonstrated that with time after grafting there is a tendency to a reduction of the process of collagen deposition and to an increase of muscle growth, especially in the presence of stimulation by angiogenic factors (15).
In any case, there is no doubt that the amount of collagen and mainly its behavior along time after surgery are important for the analysis of tissue response to the graft used. The present study using a latex biomembrane demonstrated that, despite the greater amount of collagen in the graft area compared to the native bladder during the initial period of observation (Group A), there was a progressive reduction of collagen negatively and linearly correlated with time. These results reduce the possibility that the use of a biomembrane graft will result in an exclusive healing process with fibrosis, rather indicating the occurrence of tissue remodeling and regeneration.

The angiogenesis process may occur by migration of endothelial progenitor cells (EPCs) or by capillary growth of preexisting vessels. Despite a variety of factors that may participate in various stages of angiogenesis, VEGF is one of the most important factors in adult tissues submitted to physiological angiogenesis (proliferative endometrium) and to pathological angiogenesis (inflammation, scarring and tissue repair).

The action of VEGF in the improvement of the tissue repair process results from two major characteristics, i.e., an increased capillary permeability that permits the arrival of inflammatory cells at the site of injury, and a more intense migration and proliferation of preexisting endothelial cells (16).

For the appropriate development of native tissue, the biodegradable molds must be practically impermeable to urine on their luminal surface and should permit cellular and vascular growth in the portion in contact with native tissue, an essential factor for cell survival (17). The neovascularization of regenerated tissues is of great importance since tissues do not grow more than a few millimeters unless they are vascularized (13). A study on humans using a graft with a seeding technique for bladder expansion has emphasized that neovascularization is one of the fundamental factors for appropriate cell growth in a biodegradable graft (18).

In the present study, there was a significant amount of vessels in the neoformed tissue, especially during the early phases of the process of tissue repair (first 15 days). A study using bladder regeneration with an acellular matrix under stimulation 
with VEGF demonstrated that neovascularization is more intense during the first 4 weeks (19), a result similar to that obtained here. It is believed that rapid generation of a vascular network is imperative for providing the blood supply required for newly developed tissues (20).

The comparison of VEGF analysis among the three groups presented here demonstrated that the expression of this marker of angiogenesis was more intense in Group A (15 days), being significantly reduced in the 90 day group compared to their respective controls. On this basis, we confirmed the angiogenic potential of the membrane and demonstrated that the process is temporary, accompanying the reduction of the inflammatory process, important events for the lack of perpetuation of inadequate tissue response to the graft used. An explanation for this phenomenon could be the intense macrophage activity during the early phases of scarring (9), supporting the statement that these cells play a controlling role in fibroblast proliferation and migration, in matrix formation, and in angiogenesis $(21,22)$.

\section{CONCLUSIONS}

This study demonstrates that the latex biomembrane as a matrix for partial bladder replacement in rabbits promotes temporary collagen deposition and stimulates a angiogenic process. Future studies mainly with longer time of grafting and evaluation of bladder functional properties and the detailed immunological response should be carried out.

\section{CONFLICT OF INTEREST}

None declared.

\section{REFERENCES}

1. Higuchi TT, Granberg CF, Fox JA, Husmann DA: Augmentation cystoplasty and risk of neoplasia: fact, fiction and controversy. J Urol. 2010; 184: 2492-6.

2. Blaivas JG, Weiss JP, Desai P, Flisser AJ, Stember DS, Stahl PJ: Long-term followup of augmentation enterocystoplasty and continent diversion in patients with benign disease. J Urol. 2005; 173: 1631-4.
3. Gilbert SM, Hensle TW: Metabolic consequences and longterm complications of enterocystoplasty in children: a review. J Urol. 2005; 173: 1080-6.

4. Stanasel I, Mirzazadeh M, Smith JJ 3rd: Bladder tissue engineering. Urol Clin North Am. 2010; 37: 593-9.

5. Lindley RM, Mackinnon AE, Shipstone D, Tophill PR: Longterm outcome in bladder detrusorectomy augmentation. Eur J Pediatr Surg. 2003;13(Suppl 1): S7-12.

6. Cartwright LM, Shou Z, Yeger H, Farhat WA: Porcine bladder acellular matrix porosity: impact of hyaluronic acid and lyophilization. J Biomed Mater Res A. 2006; 77: 180-4.

7. Ayyildiz A, Akgül KT, Huri E, Nuhoğlu B, Kiliçoğlu B, Ustün H, et al.: Use of porcine small intestinal submucosa in bladder augmentation in rabbit: long-term histological outcome. ANZ J Surg. 2008; 78: 82-6.

8. Brandão ML, Coutinho-Netto J, Thomazini JÁ, Lachat JJ, Muglia VF, Piccinato CE: Prótese vascular derivada do látex. J Vasc Brás. 2007; 6: 130-41.

9. Domingos AL, Tucci S Jr, Garcia SB, de Bessa J Jr, Cologna AJ, Martins AC: Use of a latex biomembrane for bladder augmentation in a rabbit model: biocompatibility, clinical and histological outcomes. Int Braz J Urol. 2009; 35: 217-24; author reply 225-6.

10. Wefer J, Sievert KD, Schlote N, Wefer AE, Nunes L, Dahiya $\mathrm{R}$, et al.: Time dependent smooth muscle regeneration and maturation in a bladder acellular matrix graft: histological studies and in vivo functional evaluation. J Urol. 2001; 165: 1755-9.

11. O'Kane S: Wound remodelling and scarring. J Wound Care. 2002; 11: 296-9.

12. Kwon TG, Yoo JJ, Atala A: Local and systemic effects of a tissue engineered neobladder in a canine cystoplasty model. J Urol. 2008; 179: 2035-41.

13. Kajbafzadeh AM, Payabvash S, Salmasi AH, Sadeghi Z, Elmi A, Vejdani $K$, et al.: Time-dependent neovasculogenesis and regeneration of different bladder wall components in the bladder acellular matrix graft in rats. J Surg Res. 2007; 139: 189-202.

14. Sicard RE: Differential inflammatory and immunological responses in tissue regeneration and repair. Ann N Y Acad Sci. 2002; 961: 368-71.

15. Loai $\mathrm{Y}$, Yeger $\mathrm{H}, \mathrm{Coz} \mathrm{C}$, Antoon R, Islam SS, Moore K, et al.: 15- Bladder tissue engineering: tissue regeneration and neovascularization of HA-VEGF-incorporated bladder acellular constructs in mouse and porcine animal models. J Biomed Mater Res A. 2010; 94: 1205-15.

16. Galiano RD, Tepper OM, Pelo CR, Bhatt KA, Callaghan $\mathrm{M}$, Bastidas N, et al.: Topical vascular endothelial growth factor accelerates diabetic wound healing through increased angiogenesis and by mobilizing and recruiting bone marrow-derived cells. Am J Pathol. 2004; 164: 1935-47. 
17. Zhang Y, Frimberger D, Cheng EY, Lin HK, Kropp BP: Challenges in a larger bladder replacement with cell-seeded and unseeded small intestinal submucosa grafts in a subtotal cystectomy model. BJU Int. 2006; 98: 1100-5.

18. Atala A, Bauer SB, Soker S, Yoo JJ, Retik AB: Tissue-engineered autologous bladders for patients needing cystoplasty. Lancet. 2006; 367: 1241-6.

19. Youssif M, Shiina H, Urakami S, Gleason C, Nunes L, Igawa $\mathrm{M}$, et al.: Effect of vascular endothelial growth factor on regeneration of bladder acellular matrix graft: histologic and functional evaluation. Urology. 2005; 66: 201-7.
20. Nomi M, Atala A, Coppi PD, Soker S: Principals of neovascularization for tissue engineering. Mol Aspects Med. 2002 23: 463-83.

21. Sisco M, Chao JD, Kim I, Mogford JE, Mayadas TN, Mustoe TA: Delayed wound healing in Mac-1-deficient mice is associated with normal monocyte recruitment. Wound Repair Regen. 2007; 15: 566-71.

22. Ashley RA, Roth CC, Palmer BW, Kibar Y, Routh JC, Fung $\mathrm{KM}$, et al.: Regional variations in small intestinal submucosa evoke differences in inflammation with subsequent impact on tissue regeneration in the rat bladder augmentation model. BJU Int. 2010; 105: 1462-8.

\section{Correspondence address:} Dr. Silvio Tucci Junior Department of Surgery, Division of Urology Ribeirao Preto Medical School University of Sao Paulo Ribeirao Preto Avenida Bandeirantes, 3900. $9^{\circ}$ andar Ribeirao Preto, SP, 14048-900, Brazil Fax: +55 16 3633-0836 E-mail: silvio.tucci@sbu.org.br 